\title{
Similarity in expression accounts for correlated evolution of interacting proteins
}

\author{
Luke Hakes*, Simon C Lovell, Stephen G Oliver and David L Robertson
}

Address: Faculty of Life Sciences, University of Manchester, Manchester, M13 9PT, UK

Email: Luke Hakes* - Luke.hakes@postgrad.manchester.ac.uk

* Corresponding author

from BioSysBio 2007: Systems Biology, Bioinformatics and Synthetic Biology

Manchester, UK. II-13 January 2007

Published: 8 May 2007

BMC Systems Biology 2007, I (Suppl I):P59 doi:I0.I I86/I752-0509-I-SI-P59

This abstract is available from: http://www.biomedcentral.com/I752-0509//?issue=SI

(c) 2007 Hakes et al; licensee BioMed Central Ltd.

Studies of interacting proteins have found correlated evolution of the sequences of binding partners, apparently as a result of compensating mutations to maintain specificity. Here we analyse co-evolution of interacting proteins in yeast, demonstrating correlated evolution of binding partners in eukaryotes. However, detailed investigation of this apparent co-evolution, focussing on the proteins' surface and binding interface, surprisingly leads to no improvement in the correlation. Thus, we conclude that true co-evolution, as characterised by compensatory mutations between binding partners, is unlikely to be chiefly responsible for the apparent correlated evolution. Rather, we find that absolute expression level outperforms correlated evolution for predicting interacting protein partners. A correlation between sequence alignments can also be identified not only between pairs of proteins that physically interact, but also between those that are merely functionally related, i.e., within the same protein complex. Thus, the correlation between sequence alignments is most likely to be due to levels of gene expression being similar for interacting proteins, thus, influencing the rate of sequence evolution in similar ways. 